\title{
Digital PreDistortion for Concurrent Dual-Band Transmitter with a Single Feedback Path
}

\author{
Kwang-Pyo Lee, Haeng-Bok Kil, Eui-Rim Jeong* \\ Department of Information and Communication Engineering, HanBat National \\ University, Daejeon, Korea \\ kplee@hanbat.ac.kr,hngbokk@hanbat.ac.kr,erjeong@hanbat.ac.kr*
}

\begin{abstract}
A new digital pre-distortion technique to linearize power amplifiers (PAs) is being proposed for concurrent dual-band transmitters. In the considering system, dual-band signals are combined and amplified by a single wideband PA. The PA output signal is distorted by the cross-modulation between different band signals as well as their own inter-modulation. In conventional dual-band DPD techniques, two independent dualfeedback paths are required to convert the dual band PA outputs into baseband signals, respectively. However, it increases hardware complexity and expense. In this paper, we propose a new DPD method requiring only a single feedback path. In this new structure, the proposed technique first estimates the dual-band PA characteristics using the single feedback path. The DPD parameters are then extracted from the estimated PA characteristics. Computer simulation demonstrates that the proposed technique can achieve comparable performance with the conventional dual-feedback DPD, with significantly reduced hardware complexity.
\end{abstract}

Keywords: Concurrent dual-band, Digital Pre-distortion (DPD), PA (Power amplifier), Polynomial, Single feed-back

\section{Introduction}

Mobile communication systems have been evolving very fast and new wireless communication systems have been developing continuously in concordance with the needs of customers. To provide higher data rate wireless transmissions within the limited frequency resources, a promising and highly energy-efficient technique arising in recent years is the concurrent dual-band transmitting architecture [1]-[5]. In this architecture, dual-band signals are combined and amplified by a single wideband power amplifier (PA). However, compared to the single-band transmitter, dual-band signals amplified by a single PA are more severely distorted by nonlinear cross-products between different bands as well as the nonlinear self-products of each band's own signal. Those nonlinear distortions cannot be compensated by the conventional single-band digital pre-distortion (DPD) methods [6]-[7], since they do not consider the nonlinear cross-products between different bands.

In order to overcome these problems, concurrent dual-band DPD techniques have been proposed recently to solve the linearization of concurrent dual-band transmitters [8]-[12] Fig. 1 shows concurrent dual-band DPD systems, where the dual feedback-paths involve two radio frequency (RF) down converters and analog-to-digital converters (ADCs). This can compensate for the distortion in lower band (LB) and upper band (UB) separately by treating the signals at two bands as two independent paths. Since each DPD output is simultaneously generated from dual independent inputs, the DPD parameters can be extracted by using data sequences captured at the same time from the dual PA outputs. Therefore, dual-feedback paths are inevitably required, which increases system implementation cost and size. In addition, it leads to the degradation of the linearization 
performance if any mismatch and/or offset between two feedback paths exist. To relieve from this difficulty, a DPD architecture that requires only one feedback path is proposed in this paper. Specifically, the proposed technique first estimates the dual-band PA characteristics using the single feedback path. The DPD parameters are then extracted from the estimated PA characteristics. The performance of the proposed method is validated through computer simulation. According to the results, the proposed technique can achieve linearization performance similar to the conventional method using two feedback paths.

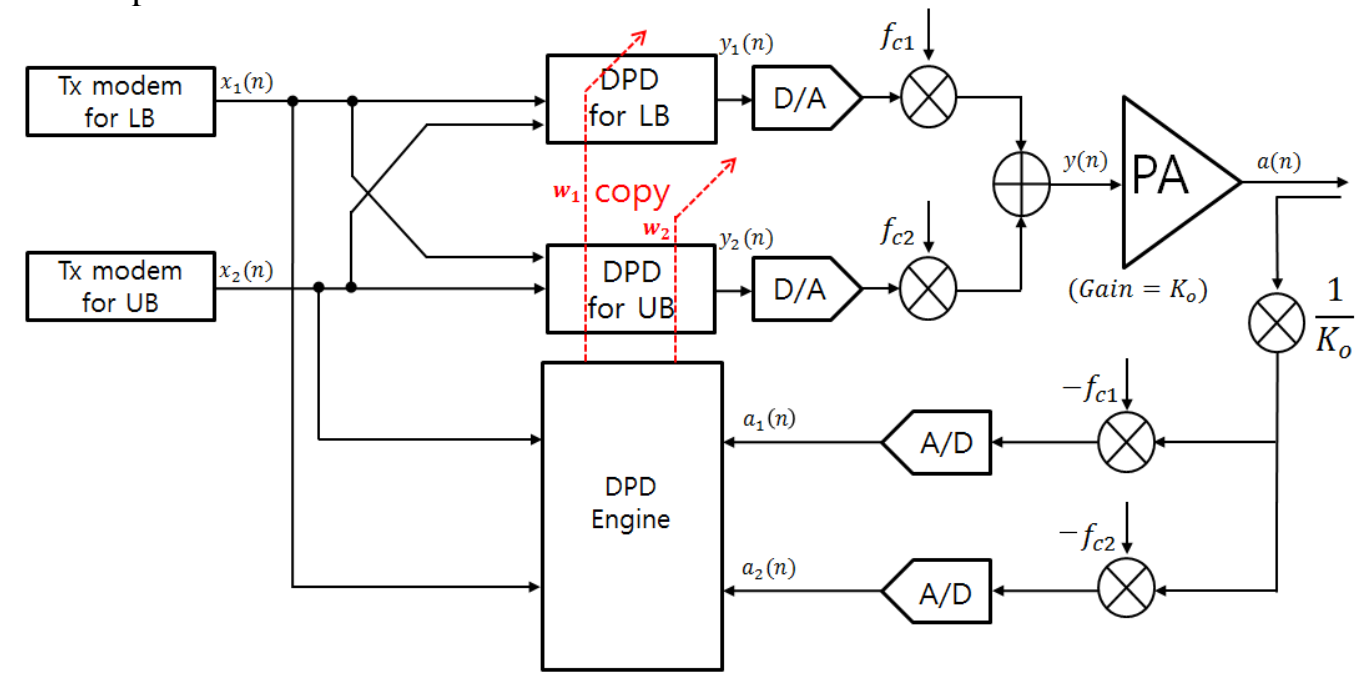

Figure 1. Conventional Architecture of Concurrent Dual-band DPD System with Dual-feedback Paths

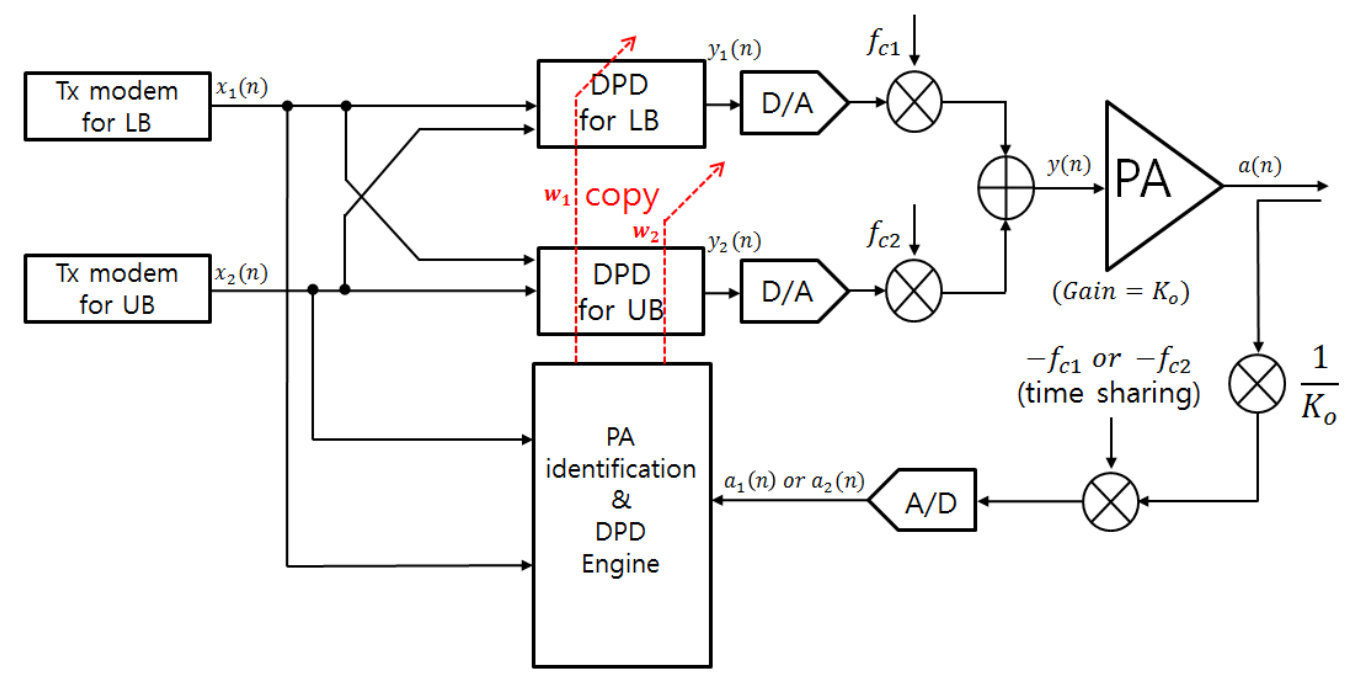

Figure 2. Proposed Architecture of Concurrent Dual-band DPD System with a Single-feedback Path

\section{System Model}

The baseband equivalent model of the proposed DPD scheme is shown in Fig 2. A concurrent dual-band transmitter with two DPDs and a single feedback path is considered. The dual streams are transmitted using dual different bands, respectively, that are not located adjacently. One DPD is for LB and the other is for UB. The outputs of both PDs are converted into analog signals by a digital-to-analog converter (DAC) and upconverted to the desired RF frequencies, respectively. Then, those up-converted signals are combined together and amplified by a single PA. To obtain DPD parameters, the 
proposed technique first estimates the dual-band PA characteristics using a single feedback path. Since there is one feedback path, PA characteristics at one of dual bands can be estimated at a time. However, by changing the local oscillator frequency at the feedback path, the other band's characteristics can be found as well. Thus, by a time sharing manner, the dual band PA's characteristics can be found sequentially. After PA identification, the DPD parameters are found from the estimated PA characteristics. This scheme reduces the number of components, cost and power consumption.

\section{Proposed Technique}

\subsection{Dual-Band DPD Structure}

For dual-band transmission with dual carriers of $f_{c 1}(\mathrm{LB})$ and $f_{c 2}(\mathrm{UB})$, the amplified PA outputs are distorted by cross-band and inter-band nonlinear products. Therefore, the input and output relation of the PA can be modeled by a nonlinear multiple input multiple output (MIMO) system [13]-[14]. The PA's input/output relations, $G_{i}(\cdot), i=1,2$, can be represented as follows:

$$
\begin{aligned}
& a_{1}(n)=G_{1}\left(y_{1}(n), y_{2}(n)\right) \\
& a_{2}(n)=G_{2}\left(y_{1}(n), y_{2}(n)\right)
\end{aligned}
$$

Let $F_{i}(\cdot), i=1,2$ be the functions representing the DPD characteristics, then DPDs functions can be written as

$$
\begin{aligned}
& y_{1}(n)=F_{1}\left(x_{1}(n), x_{2}(n)\right) \\
& y_{2}(n)=F_{2}\left(x_{1}(n), x_{2}(n)\right)
\end{aligned}
$$

where $x_{1}(n)$ and $x_{2}(n)$ are the transmitted signals at $f_{c 1}$ and $f_{c 2}$ frequencies, respectively. The ideal PD should satisfy the following relations to compensate for the nonlinearity of PA.

$$
\begin{aligned}
& G_{1}\left(y_{1}(n), y_{2}(n)\right)=K_{o} x_{1}(n) \\
& G_{2}\left(y_{1}(n), y_{2}(n)\right)=K_{o} x_{2}(n)
\end{aligned}
$$

where $K_{o}$ is the ideal gain of the PA. The proposed DPD is found through two steps : PA identification and DPD parameter calculation.

\subsection{Identification of PA Characteristics}

In this step, baseband equivalent PA models $G_{i}(\cdot)$ for $i=1,2$ are estimated. DPD is bypassed at the PA identification process, i.e., $x_{i}(n)=y_{i}(n)$. We assume that $K_{o}=1$ hereafter. The $i$-th identified PA output can be expressed as

$$
\begin{aligned}
a_{1}^{\prime} & =G_{1}\left(x_{1}(n), x_{2}(n)\right) \\
& =\sum_{m=0}^{M} \sum_{k=1}^{K} \sum_{l=1}^{k} \boldsymbol{c}_{1_{m, k, l}}^{*} x_{1}(n-m) \times\left|x_{1}(n-m)\right|^{2(k-l)}\left|x_{2}(n-m)\right|^{2(l-1)} \\
& =\boldsymbol{c}_{1}^{H} \boldsymbol{u}_{1}(n) \\
a_{2}^{\prime} & =G_{2}\left(x_{1}(n), x_{2}(n)\right) \\
& =\sum_{m=0}^{M} \sum_{k=1}^{K} \sum_{l=1}^{k} \boldsymbol{c}_{2_{m, k}, l}^{*} x_{2}(n-m) \times\left|x_{2}(n-m)\right|^{2(k-l)}\left|x_{1}(n-m)\right|^{2(l-1)} \\
& =\boldsymbol{c}_{2}^{H} \boldsymbol{u}_{2}(n)
\end{aligned}
$$


where superscripts $(\cdot)^{*}$ denotes conjugate operation, $(\cdot)^{T}$ and $(\cdot)^{H}$ denote transpose and hermitian, respectively, $2 \mathrm{~K}-1$ is the maximum polynomial order of PA, M is the memory depth and $\boldsymbol{c}_{i}$ is the weight vector of for $i$-th PA. As an example, assume that $\mathrm{K}=3$ and $\mathrm{M}=0$. Then, $\boldsymbol{c}_{1}$ and $\boldsymbol{c}_{2}$ are $6 \times 1$ weight vectors and $\boldsymbol{u}_{1}(n), \boldsymbol{u}_{2}(n)$ are written as

$$
\begin{aligned}
& \boldsymbol{u}_{1}(n)=\left[\begin{array}{c}
x_{1}(n), \\
\left.x_{1}(n)\left|x_{1}(n)\right|^{2}, x_{1}(n)\left|x_{2}(n)\right|^{2}, x_{1}(n)\left|x_{1}(n)\right|^{4},\right]^{T} \\
x_{1}(n)\left|x_{1}(n)\right|^{2}\left|x_{2}(n)\right|^{2}, x_{1}(n)\left|x_{2}(n)\right|^{4}
\end{array}\right]^{T} \\
& \boldsymbol{u}_{2}(n)=\left[\begin{array}{c}
x_{2}(n), \\
x_{2}(n)\left|x_{2}(n)\right|^{2}, x_{2}(n)\left|x_{1}(n)\right|^{2}, x_{2}(n)\left|x_{2}(n)\right|^{4}, \\
x_{2}(n)\left|x_{2}(n)\right|^{2}\left|x_{1}(n)\right|^{2}, x_{2}(n)\left|x_{1}(n)\right|^{4}
\end{array}\right]^{T}
\end{aligned}
$$

To estimate each PA, the least squares (LS) criterion is used. The LS cost function for the $i$-th PA is defined as

$$
\epsilon_{\boldsymbol{c}_{i}}=\left\|e_{\boldsymbol{c}_{i}}\right\|^{2}=\sum_{n=1}^{N}\left|a_{i}(n)-\boldsymbol{c}_{i}^{H}(n) \boldsymbol{u}_{i}(n)\right|^{2} \quad \text { for } i=1,2
$$

The solution can be found via recursive least squares (RLS) algorithm [15], summarized in Table $1 . \mathrm{N}$ is the training length.

Table 1. RLS Algorithm

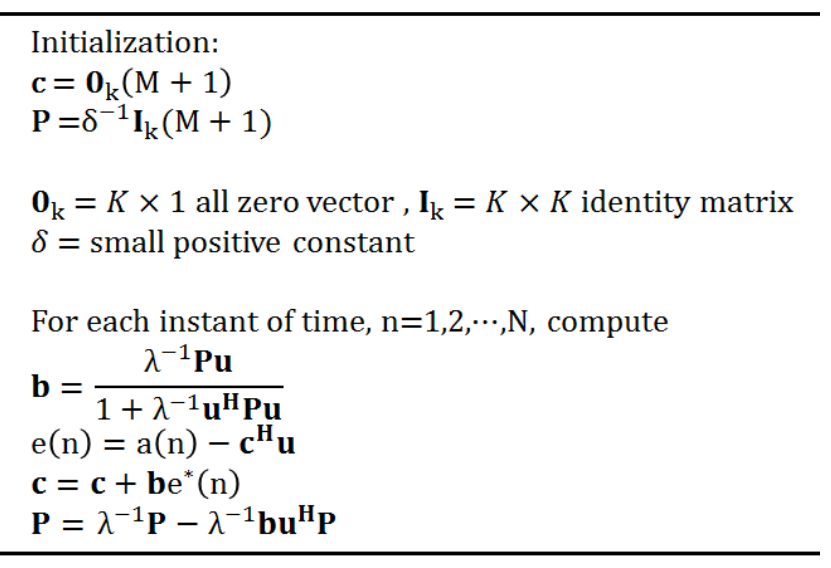

\subsection{Proposed DPD}

After finding two PA parameters, $\boldsymbol{c}_{1}$ and $\boldsymbol{c}_{2}$, we can obtain DPD parameters that linearize cross-modulation between two band signals from the identified PA model. Similarly to PA identification, the polynomial-based DPD is employed, and its parameters are estimated by using identified PA as shown in Fig 3 . Let $F_{i}(\cdot)$ denote DPD's characteristics at $i$-th band. Then the DPD's output can be written as follows:

$$
\begin{aligned}
y_{1}(n) & =F_{1}\left(x_{1}(n), x_{2}(n)\right) \\
& =\sum_{m=0}^{M} \sum_{q=1}^{Q} \sum_{l=1}^{q} \boldsymbol{w}_{1_{m, q, l}}^{*} x_{1}(n-m) \times\left|x_{1}(n-m)\right|^{2(q-l)}\left|x_{2}(n-m)\right|^{2(l-1)} \\
& =\boldsymbol{w}_{1}^{H} \boldsymbol{u}_{1}(\boldsymbol{n}) \\
y_{2}(n) & =F_{2}\left(x_{1}(n), x_{2}(n)\right) \\
& =\sum_{m=0}^{M} \sum_{q=1}^{Q} \sum_{l=1}^{q} \boldsymbol{w}_{2_{m, q}, l}^{*} x_{2}(n-m) \times\left|x_{2}(n-m)\right|^{2(q-l)}\left|x_{1}(n-m)\right|^{2(l-1)}
\end{aligned}
$$




$$
=\boldsymbol{w}_{2}^{H} \boldsymbol{u}_{2}(\boldsymbol{n})
$$

where $\mathrm{Q}$ is the maximal polynomial order of DPD. For example, assume that $\mathrm{Q}=3$ and $\mathrm{M}=0$. Then, $\boldsymbol{w}_{1}$ and $\boldsymbol{w}_{2}$ are $6 \times 1$ weight vectors and $\boldsymbol{u}_{1}(n), \boldsymbol{u}_{2}(n)$ are given by (5). Then, the LS cost function for DPD at the $i$-th Band is defined as

$$
\epsilon_{\boldsymbol{w}_{i}}=\left\|e_{\boldsymbol{w}_{i}}\right\|^{2}=\sum_{n=1}^{N}\left|x_{i}(n)-\boldsymbol{w}_{i}^{\boldsymbol{H}} \boldsymbol{v}_{i}(n)\right|^{2}(\text { for } i=1,2)
$$

where $\boldsymbol{v}_{i}$ are given by

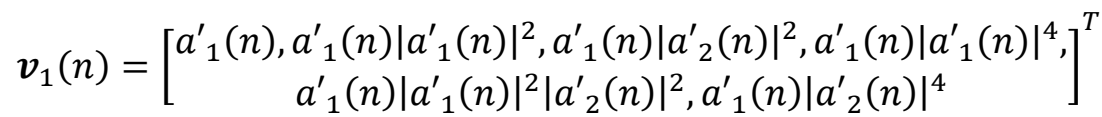

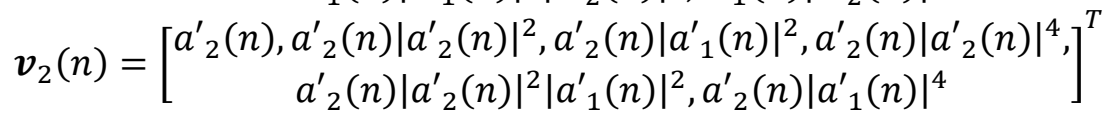

The RLS adaptive algorithm is used again for finding DPDs [15]. Therefore, We can reuse Table 1 by swapping the input and output variables, or use by replacing $x(n)$ with $a(n), \boldsymbol{c}$ with $\boldsymbol{w}$ and $\boldsymbol{u}(n)$ with $\boldsymbol{v}(n)$.

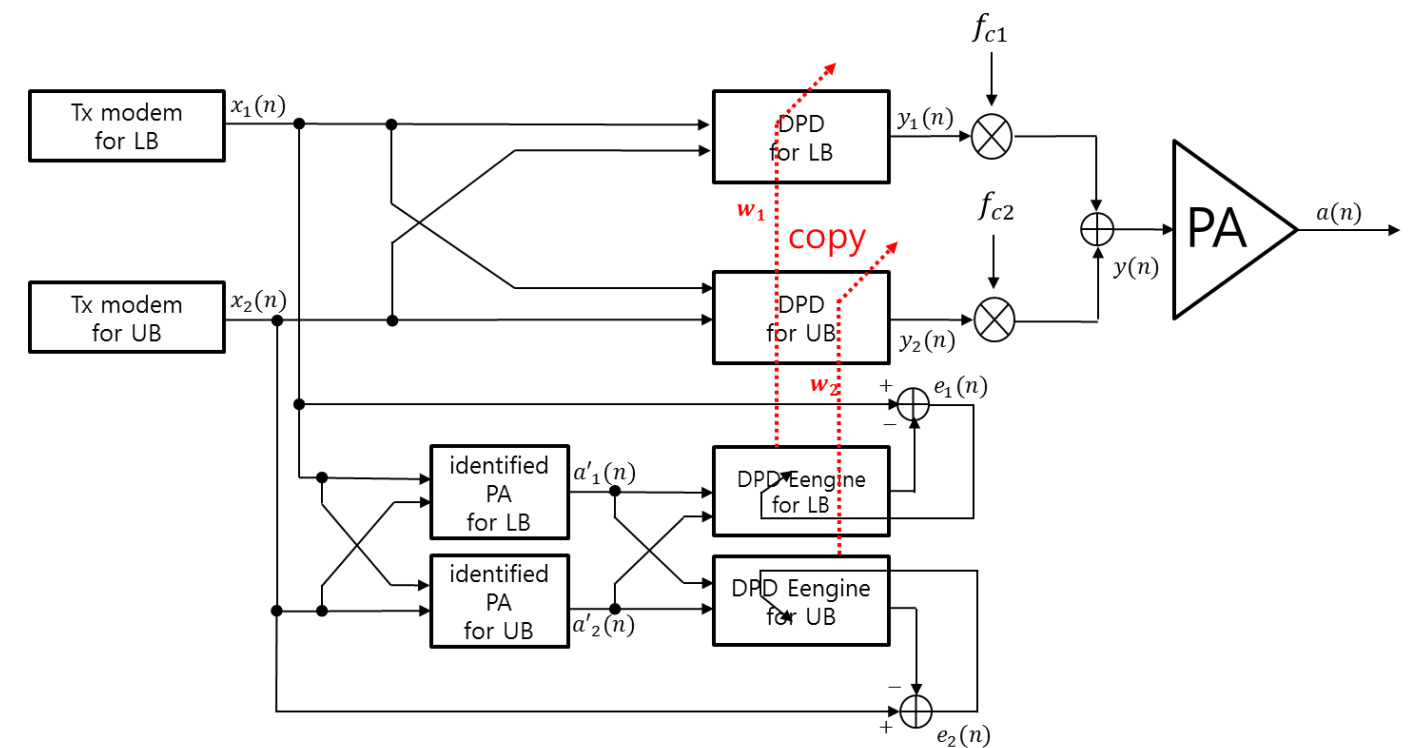

Figure 3. Detailed Diagram of the Proposed DPD Architecture after PA Identification Process

\section{Simulations Results}

\subsection{Simulation Environment}

To demonstrate the performance of the proposed method, simulation was carried out by using MATLAB. The dual-band LTE down-link signals of $20 \mathrm{MHz}$ bandwidth, centered at $800 \mathrm{MHz}$ and $1.8 \mathrm{GHz}$ are used. For the nonlinear memoryPA model, Saleh's model is employed [16]: 


$$
\begin{aligned}
G(\mathrm{y}(\mathrm{n})) & =y(n) \times \frac{K_{1}}{1+K_{2}|y(n)|^{2}} e^{\frac{K_{3}|y(n)|^{2}}{1+K_{4}|y(n)|^{2}}} \\
K_{1} & =1.1, K_{2}=0.3, K_{3}=1, K_{4}=1
\end{aligned}
$$

The memory effects are modeled by using a finite impulse response (FIR) filter with coefficients [0.7692 0.15380 .0769$]$. The DPD parameters are as follows. The maximum polynomial order of $\mathrm{PA}$ and $\mathrm{PD}$ are 5 and memory depth is $3(\mathrm{~K}=3, \mathrm{Q}=3$, $M=2)$. The forgetting factor $\lambda$ for the RLS algorithm is 0.99 .

\subsection{Performance of PA Identification}

In this section, the PA identification performance is examined. The adaptive algorithm in Table 1 is used for PA identification. The initial conditions for the parameters $\boldsymbol{c}_{i}$ are $[1,0,0, \cdots, 0]^{T}$. Learning curves for the mean square errors (MSEs) are shown in Fig 4. According to the results, the adaptive algorithm converges within 1,000 samples, and the MSE after convergence is around $10^{-10}$. The final PA model parameters are extracted as follows:

$$
\begin{aligned}
\boldsymbol{c}_{1}= & {[1.0127+0.0282 i,-0.1845-0.5808 i,-0.0753+0.3590 i,-0.2727-0.9341 i, 0.0641} \\
& +0.4361 i, 0.0023+1.2699 i, 0.0102+0.0397 i, 0.0405-0.0314 i, 0.0095-0.0361 i, \\
& 0.014-0.0008 i,-0.2676+0.2952 i,-0.5151+0.3859 i,-0.0062-0.0101 i,-0.0262+ \\
& 0.0246 i, 0.02590-0.0305 i,-0.0003-0.0071 i, 0.0767-0.0981 i, 0.2823-0.1854 i] \\
\boldsymbol{c}_{2}= & {[1.0203+0.0706 i,-0.1207-0.4673 i,-0.0248+0.1086 i,-0.3042-1.2266 i,-0.2401} \\
& +1.0987 i,-0.51859+1.7537 i,-0.0017-0.0042 i,-0.0147-0.0038 i,-0.0019+ \\
& 0.1398 i,-0.03801+0.0565 i, 0.1221-0.2259 i, 0.4107-0.3905 i,-0.0005+0.0004 i, \\
& 0.0105+0.0017 i,-0.0055-0.075 i, 0.027-0.0327 i,-0.0771+0.1367 i,-0.2698+ \\
& 0.2407 i]
\end{aligned}
$$




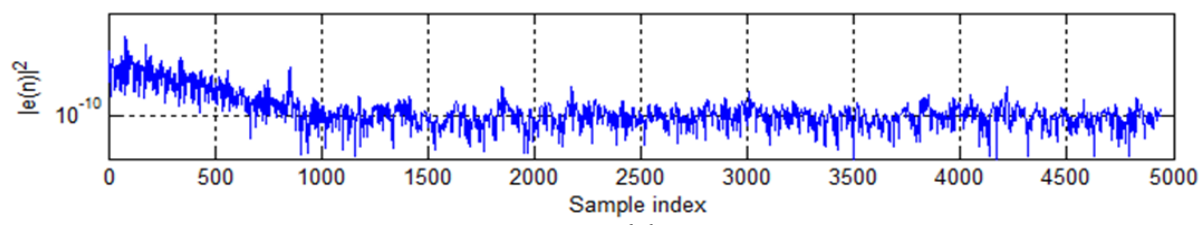

(a)

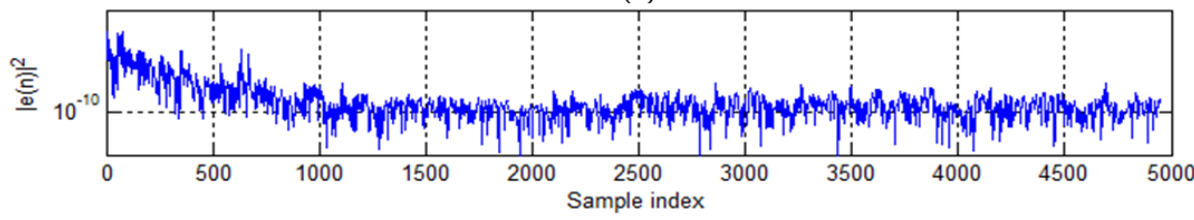

(b)

Figure 4. Learning curve (a) : LB (b) : UB
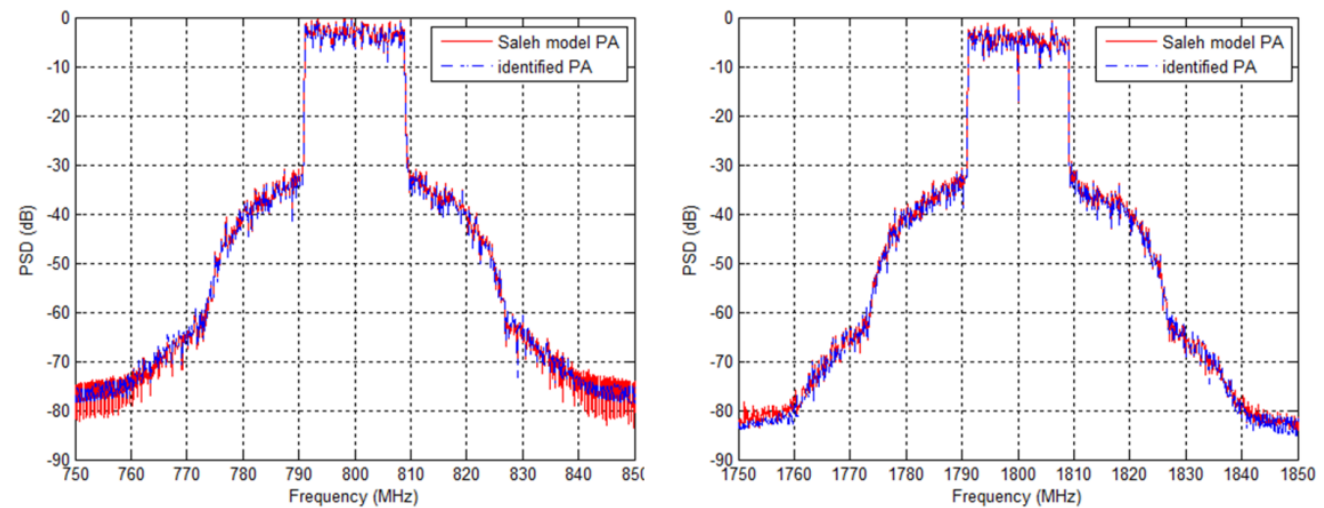

Figure 5. PA Output Spectrum (left: LB, right: UB)

To see the accuracy of the identified model, the output spectrums of the Saleh's PA output and the identified PA output are compared in Fig 5. Two spectrums almost coincide with each other. Those results indicate that the proposed PA identification method is suitable for modeling dual band PA characteristics.

\subsection{DPD performance}

After PA identification, DPD parameters are obtained. The initial condition for the DPD parameter $\boldsymbol{w}_{i}$ is $[1,0,0, \cdots, 0]^{T}$. Two learning curves for the mean square errors (MSEs) are shown in Fig 6. According to the results, the adaptive algorithms converge within 1,000 samples, and the MSE after convergence is around $10^{-11}$. The final DPD parameters are found as follows:

$$
\begin{aligned}
\boldsymbol{w}_{1}= & {[0.9871-0.0269 i, 0.2047+0.5349 i,-0.1343+0.0411 i, 0.313+0.8737 i,-0.6581} \\
& +0.1173 i,-0.6407+0.2433 i,-0.013-0.0397 i,-0.0233+0.0322 i, 0.1007-0.1151 i, \\
& -0.0139-0.0229 i, 0.5838-0.2475 i, 1.2115-0.7386 i, 0.0073+0.0103 i, 0.0286- \\
& 0.031 i,-0.0921+0.1293 i, 0.0133+0.0156 i,-0.2304+0.07 i,-0.6553+0.3888 i] \\
\boldsymbol{w}_{2}= & {[0.9813-0.0691 i, 0.1588+0.441 i,-0.0299-0.006 i, 0.4125+1.1286 i,-0.5418-} \\
& 0.1897 i,-0.0871-0.5424 i,-0.0077+0.0055 i, 0.0006-0.0217 i,-0.0248+0.003 i,- \\
& 0.0208-0.069 i, 0.0815+0.3017 i, 0.3025+0.5418 i, 0.0048-0.0012 i,-0.0007+ \\
& 0.0115 i, 0.0138+0.006 i, 0.0115+0.0422 i,-0.0566-0.1721 i,-0.176-0.2969 i]
\end{aligned}
$$

Figs.7.1 and 7.2 show the AM/AM (amplitude-to-amplitude modulation) and AM/PM (amplitude-to-phase modulation) characteristics at LB and UB, respectively. 
Red, blue and green curves represent the PA characteristics without DPD, DPD characteristics and PA characteristics with DPD, respectively. It is seen that the DPD characteristics are inverse of the PA, and the combined characteristics of the DPD and the PA are linear. For verification of signal quality at transmission signals, EVM (error vector magnitude) is evaluated. EVM is defined as follows:

$$
E V M=\sqrt{\frac{E\left[\left|x_{i}(n)-a_{i}(n) / K_{o}\right|^{2}\right]}{E\left[\left|x_{i}(n)\right|^{2}\right]}} \times 100[\%]
$$

Table 2 shows the EVM values. Without proposed DPD, the EVMs of the PA output at LB and UB are $4.046 \%$ and $4.533 \%$, respectively. Fig. 8 shows the corrupted constellations at both bands. However, after applying the proposed DPD, the EVMs at LB and UB become $0.031 \%$ and $0.054 \%$, respectively. The improved EVMs can be confirmed by constellations in Fig.8.

Fig.9 shows the power spectrums at LB and UB. Red and green colors represent the spectrums without DPD and with proposed DPD, respectively. Comparing red and green, it is verified that the proposed method reduces the spectral regrowth by $28 \sim 33 \mathrm{~dB}$ at both bands. The detailed value of the adjacent channel leakage ratio (ACLR) is listed in Table 3. This result indicates that the proposed technique can successfully mitigate the spectral regrowth with a single feedback path.

Table 2. Measured EVM performance

\begin{tabular}{|c|c|c|}
\hline & LB (\%) & UB (\%) \\
\hline Before DPD & 4.046 & 4.533 \\
\hline After DPD & 0.031 & 0.054 \\
\hline
\end{tabular}

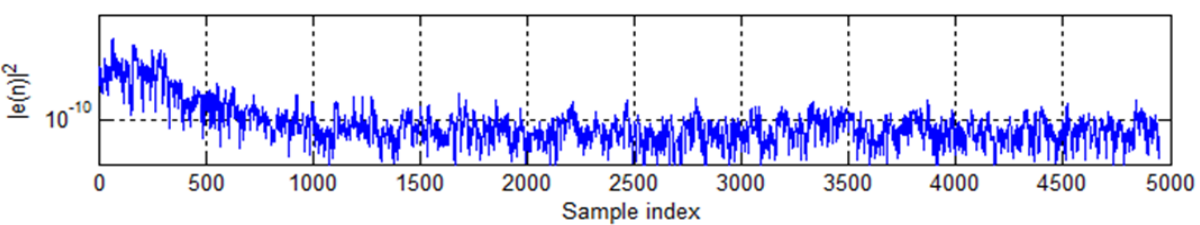

(a)

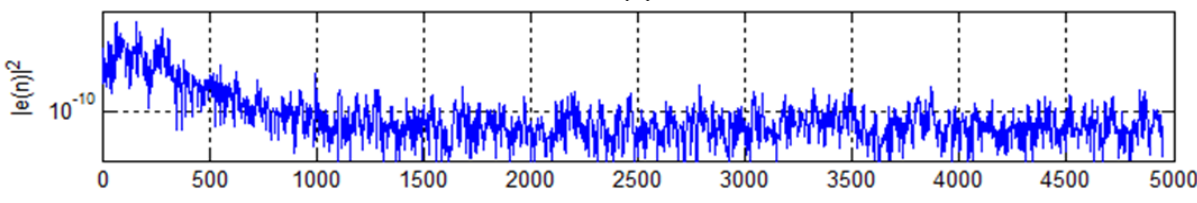

(b)

Figure 6. Learning Curve (a) : LB, (b) : UB
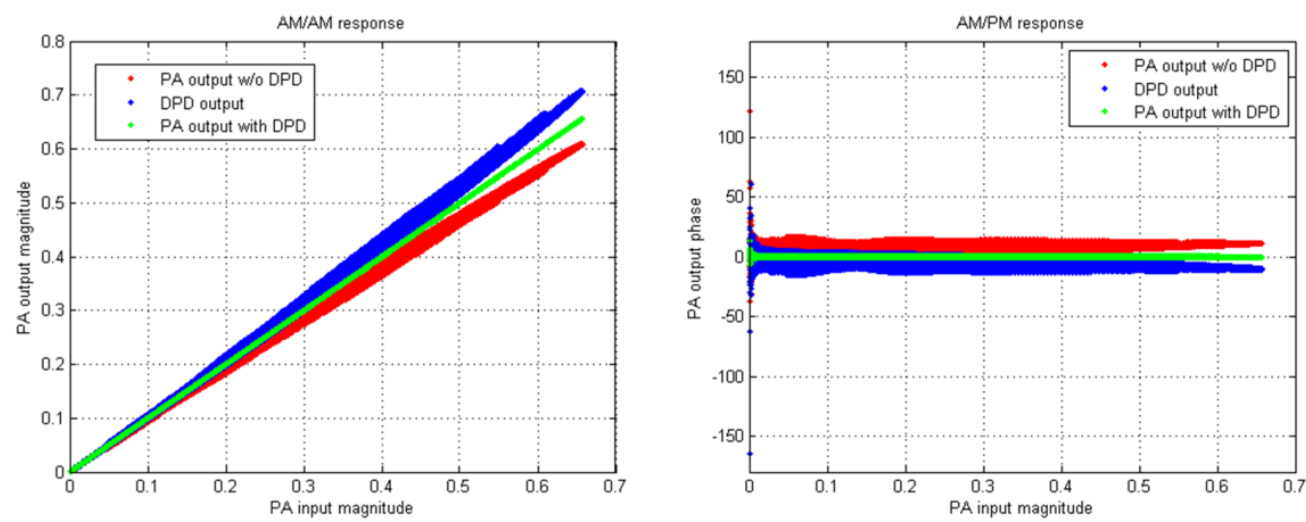

Figure 7.1 AM/AM \& AM/PM Characteristics at LB 

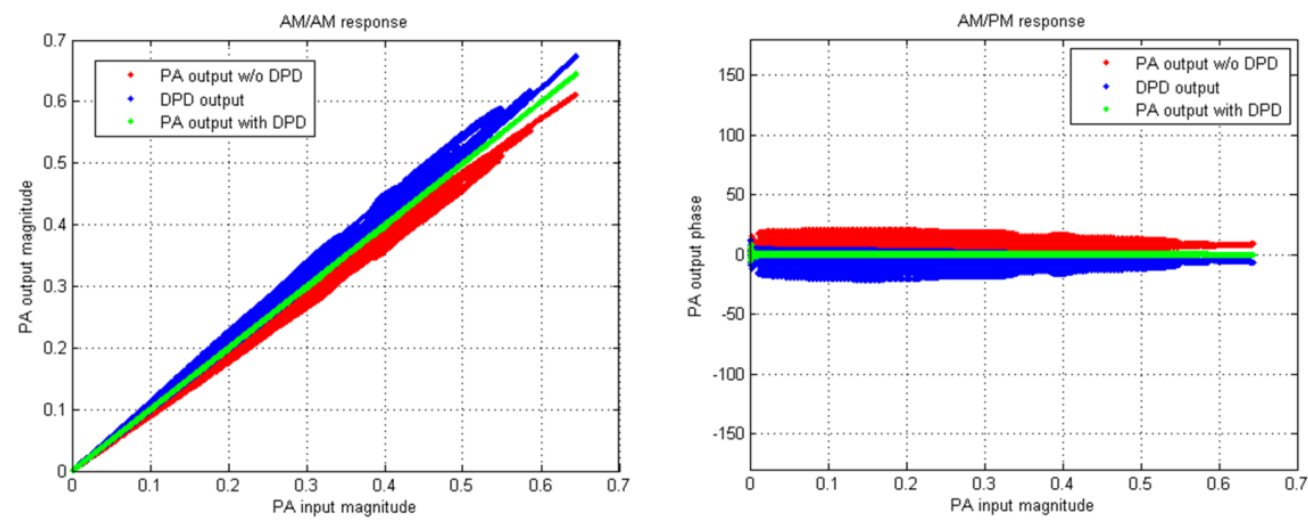

Figure 7.2 AM/AM \& AM/PM Characteristics at UB
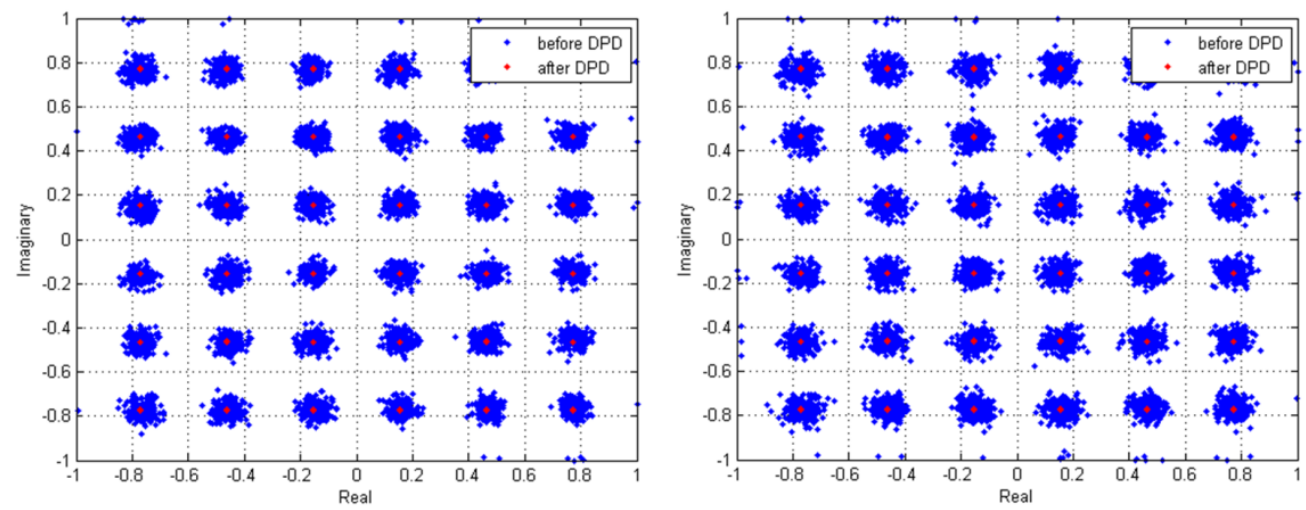

Figure 8. EVM Performance (left: LB, right: UB) 

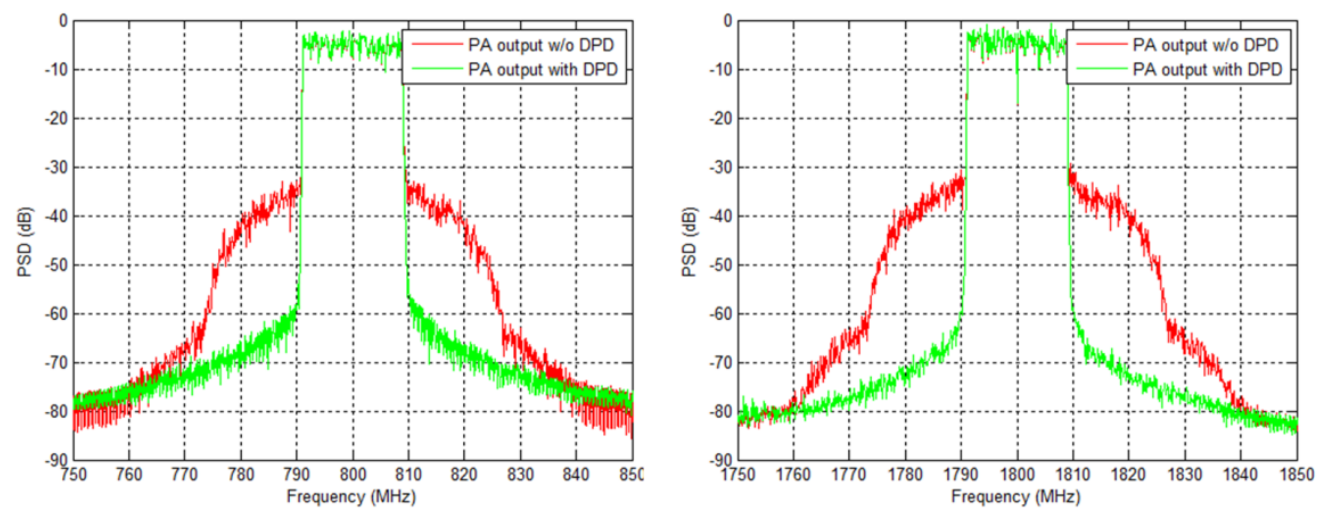

Figure 9. PA Output Spectrum with DPD (left: LB, right: UB)

Table 3. ACLR Performance

\begin{tabular}{|c|c|c|}
\hline & $\mathrm{LB}( \pm 20 \mathrm{MHz})(\mathrm{dB})$ & $\mathrm{UB}( \pm 20 \mathrm{MHz})(\mathrm{dB})$ \\
\hline Before DPD & -39.77 & -39.24 \\
\hline After DPD & -68.26 & -72.62 \\
\hline
\end{tabular}

\section{Conclusion}

A DPD technique for concurrent dual-band transmitter was proposed. Compared with conventional dual-band architecture, the proposed DPD uses only one feedback path. In this architecture, the dual band PA's characteristics can be sequentially found by changing the local oscillator frequency at the feedback path. After PA identification, the DPD parameters are found from the estimated PA characteristics. The computer simulation results verified the effectiveness of the proposed technique.

\section{Acknowledgments}

This research was supported by the World Class $300 \mathrm{R} \& \mathrm{D}$ projects funded by the Small and Medium Business Administration of Korea. (S2318109).

\section{References}

[1] Cidronali, Alessandro, et al. "Compact concurrent dual-band power amplifier for $1.9 \mathrm{GHz}$ WCDMA and $3.5 \mathrm{GHz}$ OFDM wireless systems." Microwave Integrated Circuit Conference, 2008. EuMIC 2008. European. IEEE, 2008.

[2] Simon, Martin, et al. "A high performance dual band/dual mode CMOS RF transceiver for WiMAX and WLAN systems." Wireless Technology, 2008. EuWiT 2008. European Conference on. IEEE, 2008.

[3] Negra, Renato, et al. "Concurrent dual-band class-F load coupling network for applications at 1.7 and 2.14 GHz." IEEE Transactions on Circuits and Systems II: Express Briefs 55.3 (2008): 259-263.

[4] Bassam, Seyed Aidin, et al. "Transmitter architecture for CA: Carrier aggregation in LTE-advanced systems." IEEE Microwave Magazine 14.5 (2013): 78-86.

[5] Jun Ho Jeon, Woon Geun Yang, Yeon Chan Hong. (2011.2). Design and Implementation of Internal Multi-band Folded Monopole Antenna for Mobile Station. Journal of information and communication convergence engineering, 9(1), 16-20.

[6] Lee, Kwang-Pyo, Soon-Il Hong, and Eui-Rim Jeong. "A Polynomial Digital Pre-Distortion Technique Based on Iterative Architecture." International Journal of Electrical and Computer Engineering (IJECE) 6.1 (2016): 106-112.

[7] Hong, Soon-il, Kwang-Pyo Lee, and Eui-Rim Jeong. "Robust Digital Predistortion in Saturation Region of Power Amplifiers." International Journal of Electrical and Computer Engineering (IJECE) 6.1 (2016): 99-105.

[8] Ding, Lei, Zigang Yang, and Hardik Gandhi. "Concurrent dual-band digital predistortion." Microwave Symposium Digest (MTT), 2012 IEEE MTT-S International. IEEE, 2012. 
[9] Roblin, P., Quindroit, C., Naraharisetti, N., Gheitanchi, S., \& Fitton, M. (2013). Concurrent linearization. IEEE Microw. Mag, 14(7), 75-91

[10] Bassam, Seyed Aidin, Mohamed Helaoui, and Fadhel M. Ghannouchi. "2-D digital predistortion (2-DDPD) architecture for concurrent dual-band transmitters." IEEE Transactions on Microwave Theory and Techniques 59.10 (2011): 2547-2553.

[11] Quindroit, Christophe, et al. "Concurrent dual-band digital predistortion for power amplifier based on orthogonal polynomials." Microwave Symposium Digest (IMS), 2013 IEEE MTT-S International. IEEE, 2013.

[12] Liu, You-Jiang, et al. "Digital predistortion for concurrent dual-band transmitters using 2-D modified memory polynomials." IEEE Transactions on Microwave Theory and Techniques 61.1 (2013): 281-290.

[13] Mohaisen, M. (2011). Transmit antenna selection for multi-user MIMO precoding systems with limited feedback. Journal of information and communication convergence engineering, 9(2), 193-196.

[14] S. Choi and E-R. Jeong, "Digital predistortion based on combined feedback in MIMO transmitters," IEEE Comm. Lett., vol. 16, no. 10, pp. 1572-1575, Oct. 2012.

[15] S. Haykin, Adaptive Filter Theory, 4th edition, Pearson Education, 2002.

[16] A. A. M. Saleh, "Frequency-independent and frequency-dependent nonlinear models of TWT amplifiers," IEEE Trans. Commun., vol. COM-29, no. 11, pp. 1715-1720, Nov. 1981.
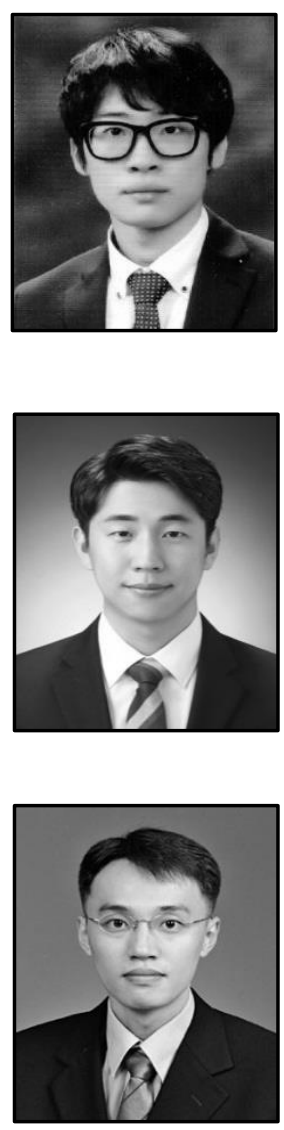

\section{Authors}

Kwang-Pyo Lee received his B.S. degree in the Department of Radio Wave Engineering from Hanbat National University, Daejeon, Korea, in 2015. He is currently pursuing his Master's degree in the Department of Radio Wave Engineering from Hanbat National University, Daejeon, Korea. His research interests are in the areas of digital signal processing, predistortion, and modem design.

Haeng-Bok Kil received his B.S. degree in the Department of Radio Wave Engineering from Hanbat National University, Daejeon, Korea, in 2016. He is currently pursuing his Master's degree in the Department of Radio Wave Engineering from Hanbat National University, Daejeon, Korea. His research interests are in the areas of digital signal processing, predistortion, and modem design.

Eui-Rim Jeong received his B.S., M.S., and Ph.D. degrees in the Department of Electrical Engineering from Korea Advanced Institute of Science and Technology (KAIST), Daejeon, Korea, in 1995, 1997, and 2001, respectively. He is currently an associate professor in department of Information and Communication Engineering, Hanbat National University, since 2009. His research interests are in the areas of communication signal processing, predistortion, and modem design. 
International Journal of Control and Automation

Vol. 10, No. 2 (2017) 